\title{
Effect of stellate ganglion block on postoperative recovery of gastrointestinal function in patients undergoing surgery with general anaesthesia: a meta-analysis
}

Bei Wen ${ }^{1}$, Yajie Wang ${ }^{2}$, Cong Zhang ${ }^{1}$ and Zhijian Fu ${ }^{1 *}$ (D)

\begin{abstract}
Background: The return of gastrointestinal function is an important sign of postoperative recovery in patients undergoing surgery with general anaesthesia. We aimed to summarize the effects of stellate ganglion block on the recovery of gastrointestinal function as a means of exploring methods through which anaesthesiologists can contribute to postoperative patient recovery.
\end{abstract}

Methods: We performed a quantitative systematic review of randomized controlled trials published between January 1, 1988, and November 11, 2019, in PubMed, the Cochrane Library, China National Knowledge Infrastructure, Chinese VIP Information, and the Wanfang and SinoMed databases. Study quality was assessed by using the GRADE criteria and bias of included studies were assessed using the revised Cochrane risk-of-bias tool for randomized trials. The time to peristaltic sound resumption, flatus, postoperative eating and the incidence of abdominal bloating in the stellate ganglion block and control groups were compared. The control group consisted of either a stellate ganglion block with normal saline or no treatment. Meta-analysis was performed using Review Manager software.

Results: After searching for relevant articles, 281 studies were identified, and five articles with data on 274 patients were eligible. Regarding postoperative flatus time, stellate ganglion block resulted in a mean reduction of $15 \mathrm{~h}$ $(P=0.02)$; then a sensitivity analysis was performed, and the standard mean difference decreased to $6 \mathrm{~h}(\mathrm{P}=0.007)$. For gastrointestinal surgery, the mean reduction was $23.92 \mathrm{~h}(\mathrm{P}=0.0002)$. As for the evaluation of the recovery of peristaltic sounds, stellate ganglion block promoted the recovery of regular peristaltic bowel sounds an average of $14.67 \mathrm{~h}$ earlier than in the control $(P=0.0008)$. When it comes to nutrients, stellate ganglion block shortened the total parenteral nutrition time by more than $50 \mathrm{~h}$ in patients who had undergone gastrointestinal surgery $(\mathrm{P}<0.00001)$. Finally, stellate ganglion block prevented the occurrence of postoperative abdominal bloating $(P=0.001)$.) No complications related to stellate ganglion block were reported.

Conclusion: Stellate ganglion block may promote postoperative gastrointestinal recovery in patients undergoing various surgeries under general anaesthesia. However, additional trials investigating the use of stellate ganglion block are necessary to confirm our finding.

\footnotetext{
*Correspondence: Zhijian_fu@163.com

1 Department of Pain Management, Shandong Provincial Hospital,

Cheeloo College of Medicine, Shandong University, 324 Jingwu Road,

Jinan 250021, Shandong, People's Republic of China

Full list of author information is available at the end of the article
}

(c) The Author(s) 2020. Open Access This article is licensed under a Creative Commons Attribution 4.0 International License, which permits use, sharing, adaptation, distribution and reproduction in any medium or format, as long as you give appropriate credit to the original author(s) and the source, provide a link to the Creative Commons licence, and indicate if changes were made. The images or other third party material in this article are included in the article's Creative Commons licence, unless indicated otherwise in a credit line to the material. If material is not included in the article's Creative Commons licence and your intended use is not permitted by statutory regulation or exceeds the permitted use, you will need to obtain permission directly from the copyright holder. To view a copy of this licence, visit http://creativecommons.org/licenses/by/4.0/. The Creative Commons Public Domain Dedication waiver (http://creativeco mmons.org/publicdomain/zero/1.0/) applies to the data made available in this article, unless otherwise stated in a credit line to the data. 
Trial registration: This meta-analysis has been registered at the International Prospective Register of Systematic Reviews (registration number CRD42020157602).

Keywords: Stellate ganglion block, Gastrointestinal function, General anaesthesia, Postoperative recovery

\section{Background}

The return of gastrointestinal (GI) function is an important sign of postoperative recovery in patients who have undergone surgery under general anaesthesia, especially in patients who receive abdominal surgery. With the emergence of Enhanced Recovery After Surgery (ERAS), the safe and effective promotion of functional GI function recovery plays an important role in rapid postoperative recovery and is an important consideration for both surgeons and anaesthesiologists.

Delayed recovery and postoperative disturbances of GI function prevent patients from resuming a normal diet and may lead to complications such as postoperative nausea and vomiting, abdominal distension and intestinal obstruction. Furthermore, it can also increase the incidence of anxiety and insomnia. These events could thus influence patients' quality of life, prolong their hospital stays, increase the associated costs, and even increase the perioperative mortality rate [1]. A decrease in GI functional recovery is mainly attributable to 3 factors. (1) The first is functional changes in the autonomic nervous system, including excitation of the sympathetic system and inhibition of the parasympathetic system. Surgical trauma and stress enhance the activity of the hypothalamic-pituitary-adrenal (HPA) axis, resulting in the release of stress hormones such as catecholamine. These stress hormones can cause vasoconstriction in the digestive tract and destruction of the protective barrier [2, 3]. (2) The destruction and injury to normal GI structures, followed by inflammation, also delay postoperative GI recovery [4]. (3) The intraoperative and postoperative use of analgesics inhibits bowel function [5]. Furthermore, opioid usage can exacerbate GI dysfunction and delay GI recovery by acting peripherally [3].

Current methods to resolve this problem are conservative and include early ambulation, reduced opioid use, administration of intravenous fluids and antiemetics, and nasogastric tube placement; however, the effects of these interventions are sometimes limited. Since delayed postoperative recovery of GI function is often driven and exacerbated by heightened sympathetic tone, for anaesthesiologists, choosing appropriate anaesthesia, maintaining proper intraoperative management and applying appropriate interventions to prevent overexcitation of the sympathetic system are vital to prompt functional GI recovery in patients.
Stellate ganglion block (SGB) is currently the most commonly used sympathetic block in medical practice; it has a wide range of indications, including complex regional pain syndrome (CRPS) types 1 and 2, postherpetic neuralgia (PHN), intractable angina, post-traumatic stress disorder (PTSD), hyperhidrosis, arrhythmias, hot flushes, cerebrovascular disease and GI dysfunction [6, 7]. It can also modify the immune response and inhibit inflammation after acute trauma $[8,9]$. Moreover, by blocking sympathetic nerves innervating the GI system, SGB can dilate GI vessels, improve the blood supply and enhance GI motility.

Based on this compelling rationale, some researchers have performed clinical trials to assess how SGB influences postoperative GI function. However, the sample sizes of these studies are relatively small, and the results are not completely consistent. It is difficult for an individual study to guide clinical practice. Thus, we sought to conduct a systematic review and meta-analysis of published studies exploring the effects of SGB on the recovery of GI function in patients undergoing surgery with general anaesthesia.

\section{Methods}

This meta-analysis was conducted according to the Preferred Reporting Items for Systematic Reviews and Meta-Analyses (PRISMA) Statement [10] and has been registered at the International Prospective Register of Systematic Reviews (registration number CRD42020157602).

\section{Searching strategy}

We searched for relevant clinical studies published between January 1, 1988 and November 11, 2019 by searching databases, including PubMed, Cochrane library, China National Knowledge Infrastructure (CNKI), Chinese VIP Information (VIP), the Wanfang and the SinoMed databases. Language was restricted to English and Chinese. All patients in these studies were adults $>18$ years of age. Combined text and $\mathrm{MeSH}$ terms were used for searching; the detailed search strategies are described in Additional file 1: Appendix A. All potentially eligible studies were considered for review irrespective of the primary outcome. Manual searches were performed using the reference lists of crucial articles. 


\section{Inclusion and exclusion criteria}

Studies were considered eligible if they were random controlled clinical trials involving SGB for patients undergoing surgery with general anaesthesia and reporting postoperative GI function such as bowel sounds, the incidence of abdominal bloating, time to flatus, and time to eating. They should also include a control group, which was defined as patients receiving SGB with saline or general anesthesia alone (i.e. no block). The exclusion criteria were as follows: (1) observational and retrospective studies; (2) studies without a control group; (3) studies that did not assess GI function; (4) a reporting language other than English or Chinese.

\section{Study selection, data extraction and quality assessment} Study selection and data extraction were performed by three authors (BW, YJW, and CZ) independently. Disagreements and difficulties were resolved by group discussion or by consultation with another author (ZJF). The titles and abstracts of all articles were first screened according to the inclusion and exclusion criteria. The full text of the article was then carefully read for final determination. If the study satisfied the inclusion criteria, it was used for detailed analysis and data extraction. The following data were extracted from the selected studies: (1) demographic data (total number of participants, age, sex); (2) treatment protocols (methods, side treated, drug category, drug dosage); (3) the outcomes related with postoperative GI recovery (postoperative recovery time of bowel sounds, the incidence of abdominal bloating, time to flatus, and time to eating); and (4) any complications related with SGB. The bias of the included studies was assessed according to the revised Cochrane risk-ofbias tool for randomized trials (Rob-2). And the quality of trials was assessed using the GRADE system.

\section{Statistical analysis}

We assessed the effect of SGB on postoperative GI recovery in terms of four outcomes: time to flatus, time to resumption of peristaltic sounds, postoperative eating, and incidence of abdominal bloating. The first of these was the primary outcome. The first three outcomes were analysed as continuous variables. The last outcome was analysed as a dichotomous variable. We reported absolute differences between patients who received different interventions and calculated pooled estimates of the mean differences in all these outcomes between the intervention groups. If the heterogeneity was low $\left(\mathrm{I}^{2}<50 \%\right)$, a fixed-effects model was used for pooled analysis; a random-effects model was used if the heterogeneity was high to account adequately for the additional uncertainty associated with the use of data from different studies.
Moreover, if the heterogeneity was high, a sensitivity analysis was performed to explore the cause of it. Publication bias was assessed using a funnel plot. We used the Cochran $\mathrm{I}^{2}$ test to assess the existence and magnitude of heterogeneity among the studies [11]. Heterogeneity was considered low, moderate, or high for $\mathrm{I}^{2}$ values $<25 \%$, $25-50 \%$, and $>50 \%$, respectively.

Review Manager (RevMan 5.3) and Stata 14.0 was used in all statistical analyses. $\mathrm{P} \leq 0.05$ was considered statistically significant.

\section{Results}

In all, 281 studies were identified after a search for relevant articles; five articles [12-16] with data on 274 patients were eligible for this analysis. Of the 276 excluded studies, 41 were removed for duplications, 215 were removed after screening of their titles and abstracts according to the inclusion criteria, 1 was removed because the patient did not receive general anaesthesia, 5 were removed because they were not human studies, 12 were removed because they were not postoperative studies, 1 was removed because it did not focus on GI function, and 1 was removed because it was published repeatedly. Figure 1 shows the process of study selection.

\section{Summary characteristics of the included studies}

The five studies included in our analysis that met the inclusion criteria but not the exclusion criteria were published between 2013 and 2019. The characteristics of the included studies are shown in Table 1, which lists the characteristics of these studies in detail, including type of operation, time and side of SGB, type and volume of local anaesthetic, and other parameters.

\section{Risk of bias of included studies}

The bias of the included studies was assessed according to the Rob-2 [17]. Figure 2 presents detailed information about this assessment. Two studies had low overall bias $[12,16]$, and three had some concerns regarding bias [13-15]. All three of these studies performed SGB before induction and relied on the observation of Horner's syndrome to ensure a successful block; thus, the care givers and those delivering the interventions might have been aware of the participants' assigned interventions. In two of the included studies, no sham procedures were performed, so the allocation concealment and blinding of participants and personnel could not be assessed [14, 15]. Figure 3 shows a funnel plot of the included studies. Because we included only 5 studies, quantitative analyses such as the Begg and Egger tests were not performed. The overall quality of our meta-analysis is shown in Table 2; the results indicate that we have a moderate overall certainty of evidence. 


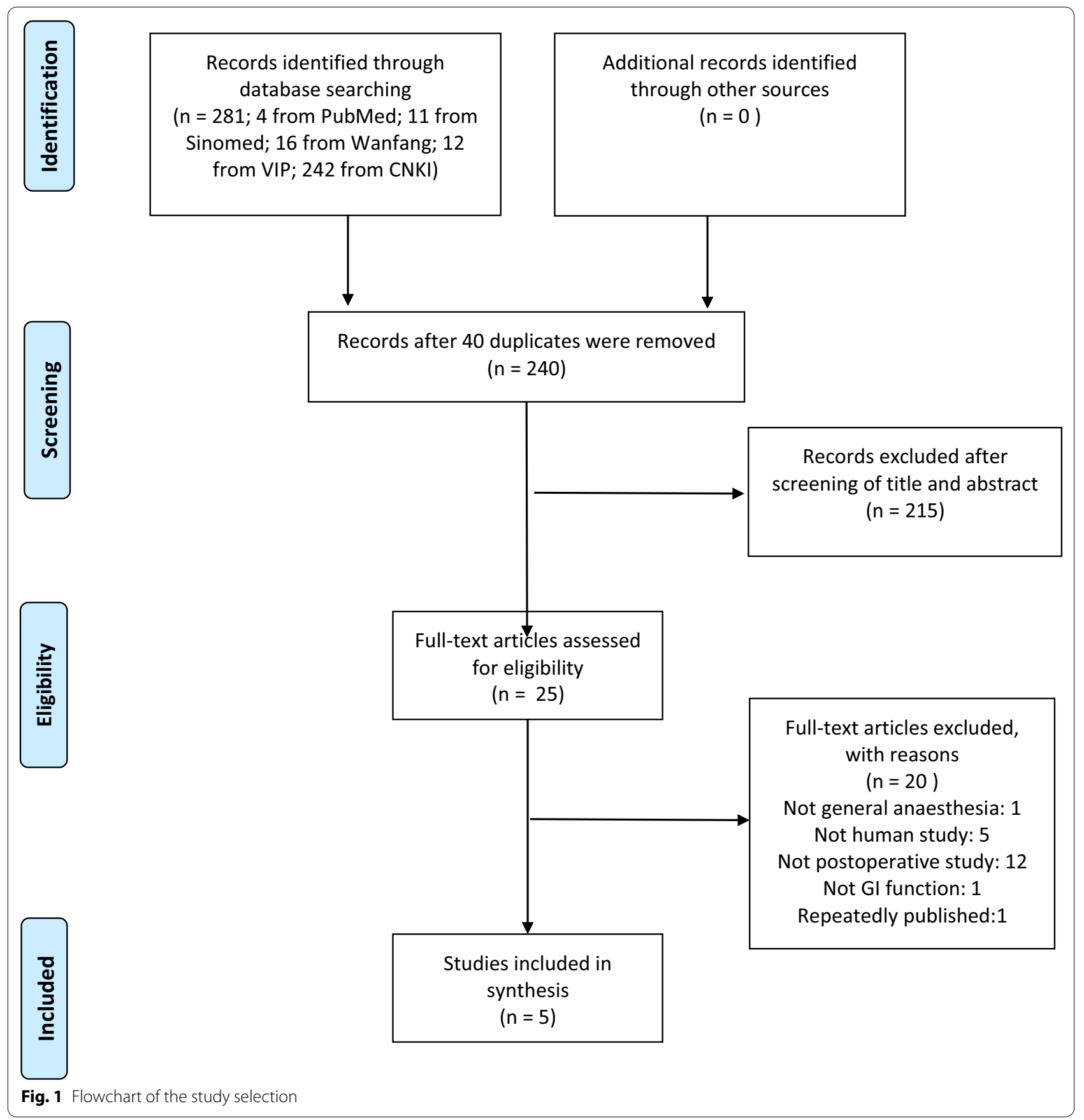

\section{Primary outcome}

Our primary outcome is comparison of the postoperative flatus time in the SGB and control groups; this comparison is shown in Fig. 4. All included studies reported this outcome, but there was great heterogeneity $\left(\mathrm{P}<0.00001, \mathrm{I}^{2}=98 \%\right)$. Our analysis showed an overall effect size (mean difference, MD) of $-15.07 \mathrm{~h}(95 \%$ $\mathrm{CI}-27.58,2.56)$ with a $\mathrm{Z}$ value of $2.36(\mathrm{P}=0.02<0.05)$. However, as shown in Fig. 5, when we deleted one particular study [15], the heterogeneity was greatly reduced $\left(\mathrm{P}=0.02<0.05, \mathrm{I}^{2}=71 \%\right)$, and the overall effect size (MD) became $-6.77 \mathrm{~h}(95 \% \mathrm{CI}$ : $-11.67,1.88)$ with a $\mathrm{Z}$ value of $2.71(\mathrm{P}=0.007<0.05)$. We think that this phenomenon was caused by the low quality of that study, as shown in Figs. 1 and 2; that study did not describe how the random sequence was generated, the SG did not receive any treatment in the control group, and the side on which SGB was performed in the SGB group was 


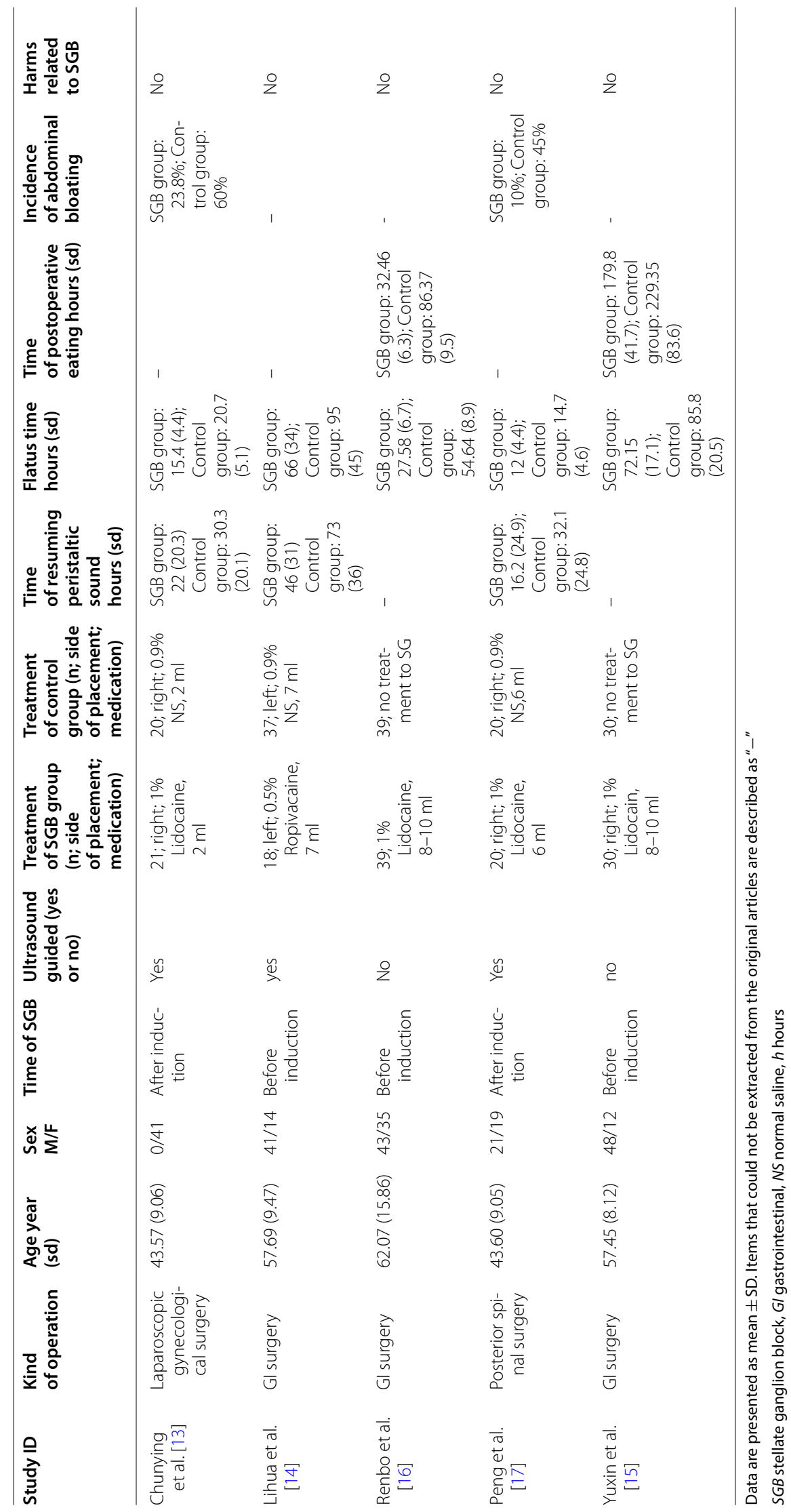




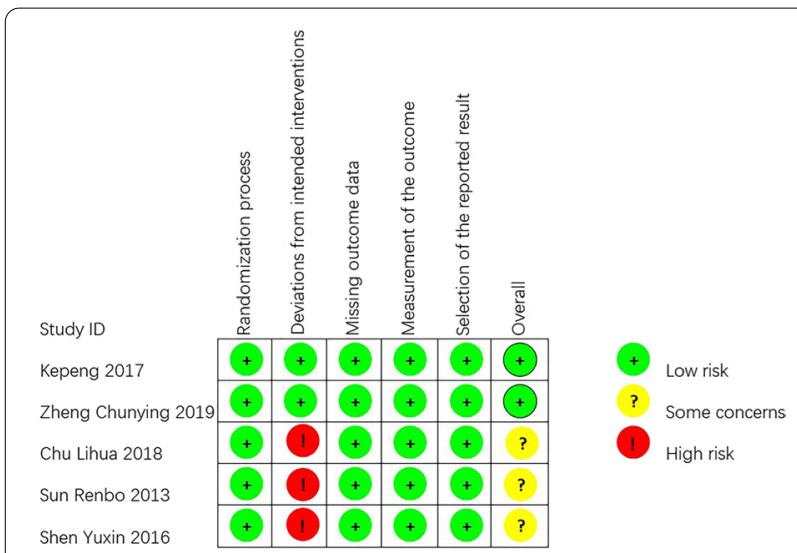

Fig. 2 Bias assessment graph. Bias were assessed by using the Rob2 tool

not reported. Furthermore, the population evaluated in this study was the oldest among the five studies, and the surgery was performed to treat GI tumours. However, despite the heterogeneity, inclusion of that study did not change the effect of SGB on the postoperative flatus time.

We also performed a subgroup analysis of 3 clinical trials in patients who underwent GI surgery surgery [1315]. In these three studies, SGB was performed before induction of general anaesthesia. As shown in Fig. 6, the overall effect size (MD) was $-23.92 \mathrm{~h}(95 \% \mathrm{CI}-36.49$, $11.35)$ with a $Z$ value of $3.73(P=0.0002<0.05)$, indicating that administration of SGB before anaesthesia significantly shortened the time to flatus after GI surgery.

Overall, our analysis suggests that SGB may shorten the postoperative flatus time in patients who undergo various types of surgery under general anaesthesia, with a mean reduction of more than $6 \mathrm{~h}$. This reduction was most obvious in patients who underwent GI surgery, with a mean reduction of $23.92 \mathrm{~h}$.

\section{Secondary outcomes}

\section{Time to resumption of peristaltic sounds}

Three of the included studies reported the time to resumption of peristaltic sounds after surgery; these studies involved patients who underwent GI, laparoscopic gynaecological and posterior spinal surgery, respectively $[12,13,16]$. However, the data were presented as the number of patients whose bowel sounds recovered within a given period of time after the operation, such as before $12 \mathrm{~h}$, before $24 \mathrm{~h}$, before $36 \mathrm{~h}$, before $48 \mathrm{~h}$, and before $72 \mathrm{~h}$ postoperatively. For convenience, we converted these data as follows: the average of the reported times was used as the time to resumption of peristaltic sounds in the patients; thus, this outcome was also analysed as continuous. Figure 7 shows a comparison of postoperative peristaltic sound resumption time in

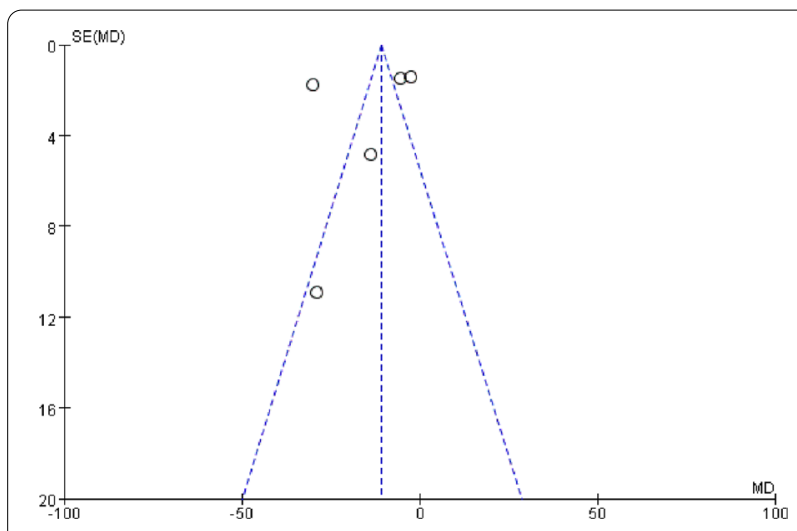

Fig. 3 Funnel plot for postoperative flatus time. SE, standard error; $\mathrm{MD}$, mean difference. The blue vertical dashed line represents the mean of MD, and the 2 blue slant dashed lines represent $95 \% \mathrm{Cl}$ of $\mathrm{MD}$

the SGB and control groups. The overall effect size (MD) was $-14.67 \mathrm{~h}(95 \% \mathrm{CI}-23.21,-6.12)$ with a $\mathrm{Z}$ value of $3.36(\mathrm{P}=0.0008<0.05)$. Furthermore, the heterogeneity was low $\left(\mathrm{P}=0.25>0.05, \mathrm{I}^{2}=28 \%\right)$, indicating that the combined analysis of these three studies is reasonable. This outcome, to some degree, may show that SGB can promote GI movement after different types of surgery in patients who receive surgeries under general anaesthesia. However, additional studies are needed to confirm this.

\section{Time to postoperative eating}

Two of the included studies reported time to postoperative eating, and the parameters in these two studies were very similar $[14,15]$. First, both studies involved patients who had undergone GI surgery. Second, SGB was performed before the induction of general anaesthesia in both studies. Third, the local anaesthetic used consisted of $8-10 \mathrm{ml}$ of $1 \%$ lidocaine in both studies. Last, neither study used a sham group, and no treatment was applied in the control group. Figure 8 shows the data for this outcome. There was no heterogeneity between these 2 studies $\left(\mathrm{P}=0.80>0.05, \mathrm{I}^{2}=0 \%\right)$. The overall effect size (MD) was $-53.86 \mathrm{~h}(95 \% \mathrm{CI}-57.43,-50.29)$ with a $\mathrm{Z}$ value of $29.60(\mathrm{P}<0.00001)$. This result suggests that in GI surgery under general anaesthesia, performing SGB before the induction of anaesthesia can significantly shorten the time to postoperative eating by more than 2 days.

\section{Incidence of postoperative abdominal bloating}

Two studies included data on the incidence of postoperative abdominal bloating $[12,16]$. One study involved laparoscopic gynaecological surgery [12], and the other involved GI surgery [16]. As shown in Fig. 9, no heterogeneity was found $\left(\mathrm{P}=0.7070>0.05, \mathrm{I}^{2}=0 \%\right)$, and odds ratio, OR) was $0.18(95 \% \mathrm{CI} 0.06,0.51)$ with a $\mathrm{Z}$ value 


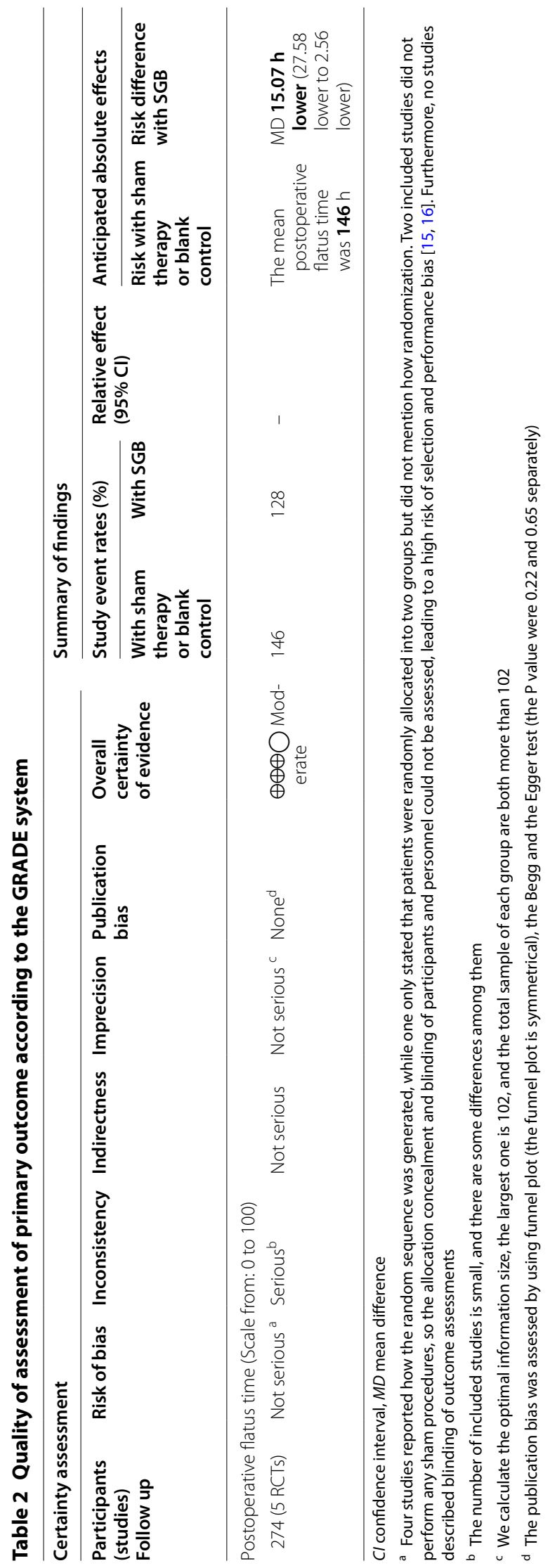




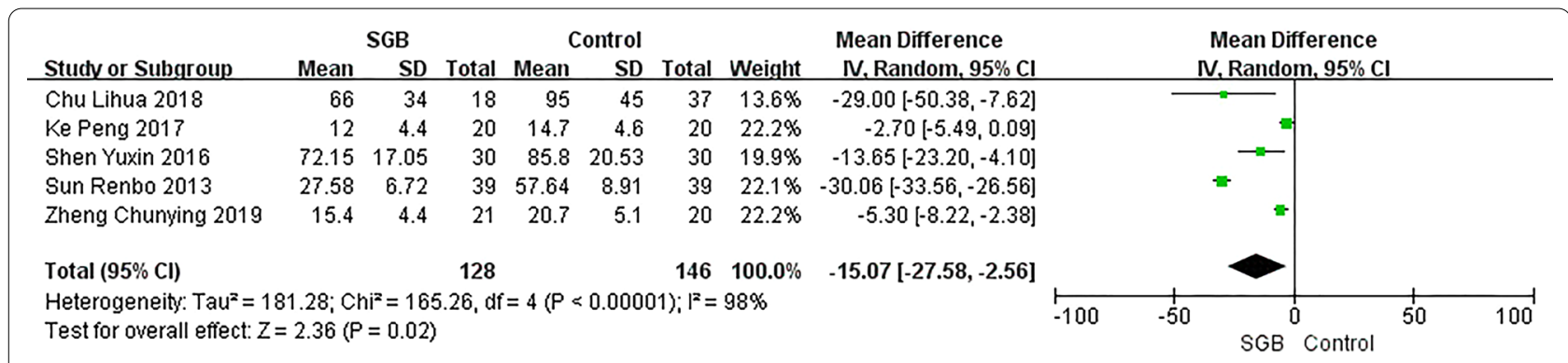

Fig. 4 Postoperative flatus time. Cl confidence interval, SD standard error, SGB stellate ganglion block. Postoperative flatus time in patients receiving SGB with local anesthetics compared to control in different surgeries

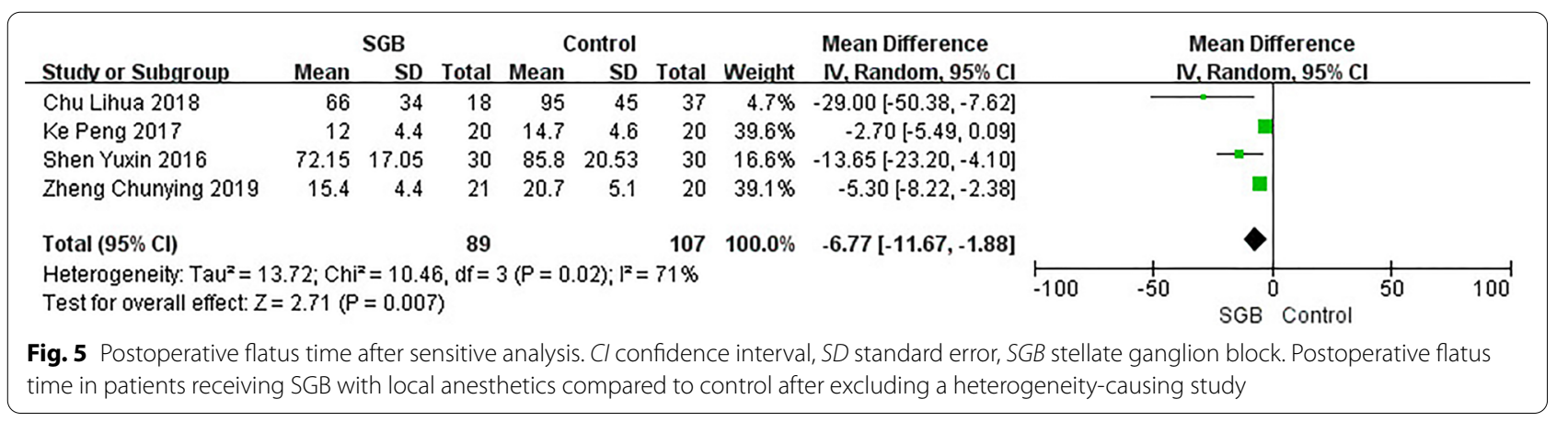

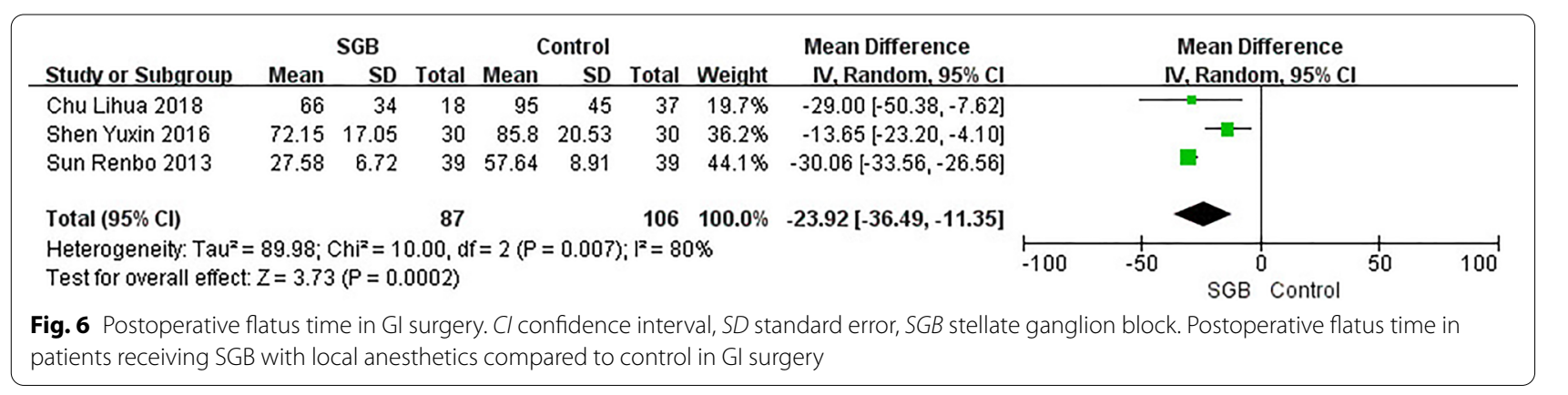

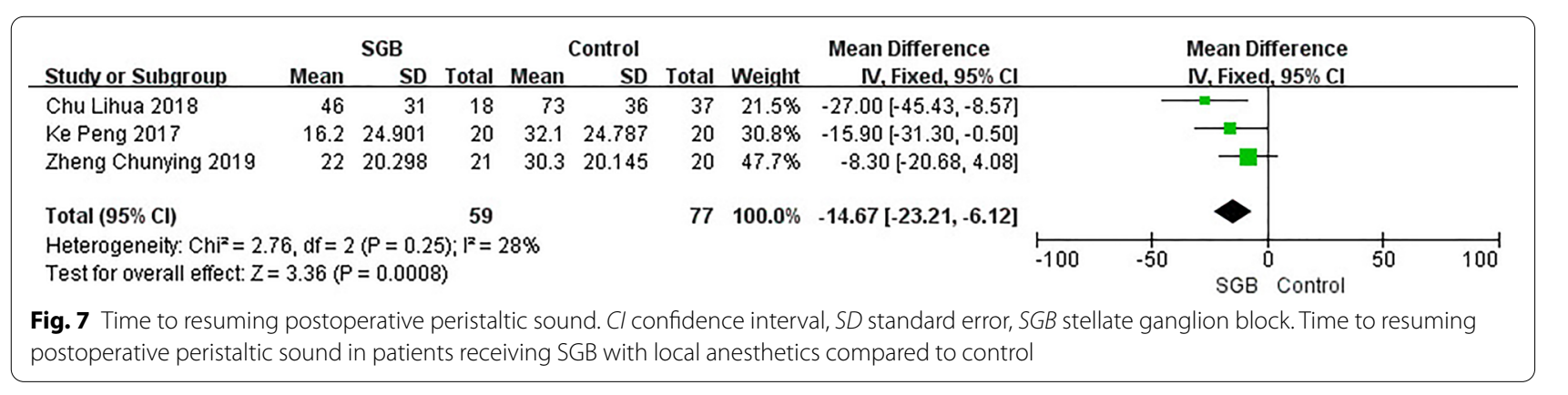

of $3.22(\mathrm{P}=0.001<0.05)$. The result indicates that SGB may reduce the incidence of postoperative abdominal bloating.

\section{Discussion}

With the continuously increasing incidence of diseases with surgical indications, the number of patients who require general anaesthesia is also increasing. Both 


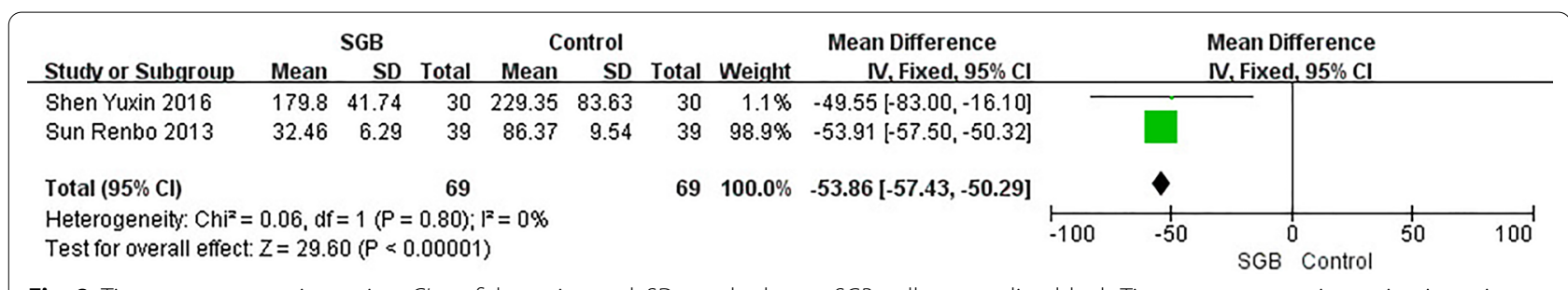

Fig. 8 Time to postoperative eating. Cl confidence interval, SD standard error, SGB stellate ganglion block. Time to postoperative eating in patients receiving SGB with local anesthetics compared to control

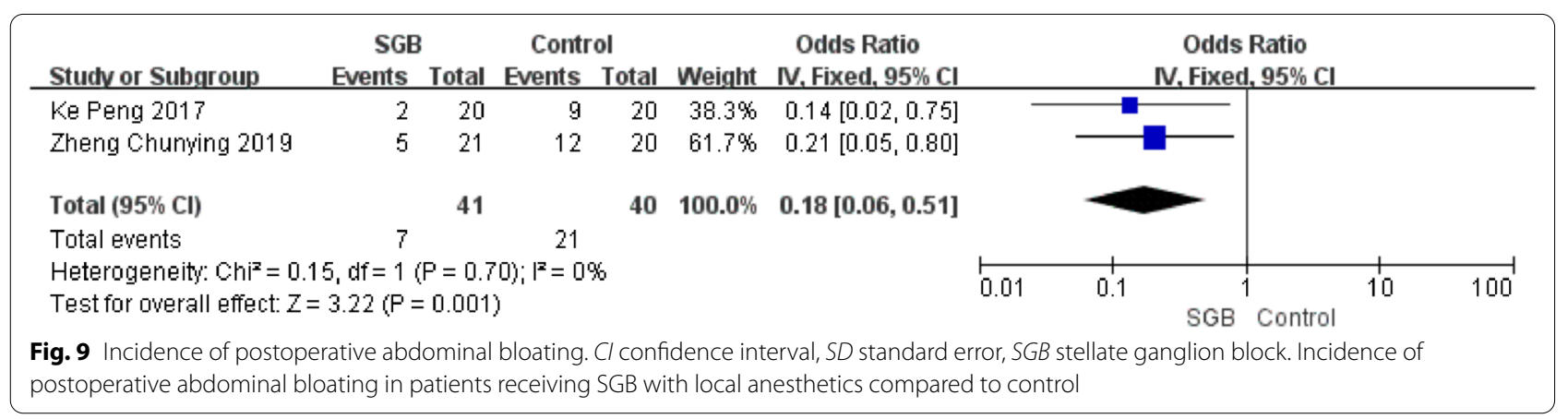

surgical manipulation and analgesia can lead to postoperative GI dysfunction. Normally, after GI surgery, gastric motility recovers in $24-48 \mathrm{~h}$, small intestinal motility recovers in $12-24 \mathrm{~h}$, and colonic motility recovers in 3-5 days [18]. The inhibition of GI function can bring about GI dysfunction and discomfort; more seriously, it could lead to systematic inflammation and even to multiple organ dysfunction syndrome [19].

There have been many studies aiming to explore methods to facilitate postoperative GI recovery. These studies have explored the following methods: (1) multimodal analgesia to reduce the use of opioids, e.g., other analgesic methods and the use of nonsteroidal anti-inflammatory drugs (NSAIDs) [20, 21]; (2) laparoscopic surgery [22]; (3) goal-directed fluid therapy [22]; (4) early enteral nutrition [23]; (5) gum chewing [24]; (6) the use of opioid receptor antagonists [25]; and (7) traditional Chinese medicine [26]. All of these methods have been shown to have limited effects in postoperative GI function recovery.

To determine whether SGB plays a role in postoperative GI function, we performed the meta-analysis reported in this paper. Although the included studies involved patients who underwent various types of surgery, the results nevertheless suggest that SGB may promote postoperative GI recovery in patients who underwent surgery with general anaesthesia. Our results show that SGB caused a mean reduction of $15 \mathrm{~h}$ in the time to flatus after different surgeries; after a study that caused heterogeneity was excluded, the mean reduction was still greater than $6 \mathrm{~h}$. Further analysis of only patients who underwent GI surgery showed a mean reduction of $23.92 \mathrm{~h}$. The data on the recovery of peristaltic sounds suggested that in patients who receive various types of surgeries, SGB promotes the recovery of regular bowel sounds $14.67 \mathrm{~h}$ earlier, on average, than the time observed for the control group. Regarding nutrients, the use of SGB in GI surgery can shorten the time for which total parenteral nutrition is required by more than $50 \mathrm{~h}$. Furthermore, our study shows that SGB can prevent the occurrence of postoperative abdominal bloating. None of the included studies reported complications related to SGB.

The role played by SGB on postoperative recovery might be explained by the following mechanism. The digestive system is mainly governed by the autonomic nervous system. As a major stressor, undergoing surgery with general anaesthesia leads to functional changes in this system, causing stimulation of the sympathetic system, inhibition of the parasympathetic system and the release of catecholamines. Blockage of cervical sympathetic nerves can suppress the overexciting of the sympathetic nervous system, help to establish balance of the autonomic nervous system and promote the establishment of homeostasis via regulation of the neuroendocrine-immune system [27], thus promote postoperative recovery of GI function.

As the most commonly used cervical ganglion block, SGB can be applied with or without imaging guidance through computed tomography or ultrasound [28, 
29]. Manifestations including Horner's syndrome, an increase in skin temperature, loss of the galvanic skin response and an increase in blood flow in the innervated areas, indicate success of the block. With the popularization of ultrasound, performing SGB under ultrasound guidance, which provides direct visualization of soft tissue structures around the sympathetic chain, appears to offer increased safety and efficacy [28]. This allows more effective and precise needle placement using a small volume of drugs. Administration of a series of local anaesthetics such as lidocaine, bupivacaine and ropivacaine can be chosen for reversible blockade. Moreover, neurolytic agents such as alcohol can be used for permanent blockade. If SGB is performed under the guidance of ultrasound, $2-5 \mathrm{ml}$ of drug is required to ensure that the procedure is as selective for sympathetic block as possible [28]. When ultrasound guidance is not possible, it has been reported that 6 to $10 \mathrm{ml}$ of drug is needed to achieve a successful blockade [30-32]. SGB was performed after and before the induction of anaesthesia in two and three studies, respectively. Performing SGB before induction can provide visible evidence of the success of the block, for example if the patient presents Horner's syndrome, but prevents blinding of the patients and doctors, while performing SGB after induction allows blinding but increases the difficulty of assessing the block's efficacy. However, if the operator is skilled or can perform SGB under ultrasound guidance, performing SGB after induction is theoretically a good choice.

There are some limitations to our study. First, only 5 studies were included in our analysis, limiting the subgroup analysis. These five studies involved patients who underwent 3 different types of surgery, and each type of surgery was addressed in only 1 or 3 studies; thus, additional studies should be performed to validate our conclusions. Second, all of the clinical trials included in this study were conducted in the People's Republic of China, restricting the generalizability of our conclusions. Moreover, SGB is an invasive treatment, and success of the block needs to be verified by some manifestations, making blinding impossible.

\section{Conclusion}

The quantitative analysis presented here shows that SGB may be moderately effective in promoting GI recovery in patients who have undergone surgery. It could reduce postoperative flatus time, promote the recovery of peristaltic sounds, shorten the total parenteral nutrition time after GI surgery and prevent the occurrence of postoperative abdominal bloating. Further clinical trials of high quality and the use of SGB in other types of surgery and in other countries are needed to confirm these results.

\section{Supplementary information}

Supplementary information accompanies this paper at https://doi. org/10.1186/s12893-020-00943-0.

Additional file 1: Searching strategy in PubMed database.

\section{Abbreviations}

SGB: Stellate ganglion block; Gl: Gastrointestinal; ERAS: Enhanced recovery after surgery; SG: Stellate ganglion; CRPS: Complex regional pain syndrome; PHN: Postherpetic neuralgia; PTSD: Post-traumatic stress disorder; PRISMA: Systematic reviews and meta-analyses; CNKI: China National Knowledge Infrastructure; VIP: Chinese VIP Information.

\section{Acknowledgements}

We thank AJE for revising the English language used in this paper.

\section{Authors' contributions}

BW and ZF designed the study, BW, YW and CZ searched and screened the literature, BW and YW were responsible for the data extraction and analysis and wrote the first draft of the manuscript, and ZF had primary responsibility for the article's final content. All authors read and approved the final manuscript.

\section{Funding}

This article is founded by grants from the National Natural Science Foundation of China (Grant Nos. 81771199; 81271346). The funding body played no role in the design of the study and collection, analysis, and interpretation of data and in writing the manuscript.

\section{Availability of data and materials}

All data generated or analysed during this study are included in this published article.

Ethics approval and consent to participate

Not applicable.

\section{Consent for publication}

Not applicable.

\section{Competing interests}

The authors declare that they have no competing interests.

\section{Author details}

${ }^{1}$ Department of Pain Management, Shandong Provincial Hospital, Cheeloo College of Medicine, Shandong University, 324 Jingwu Road, Jinan 250021, Shandong, People's Republic of China. ${ }^{2}$ Department of Gastrointestinal Surgery, Shandong Provincial Hospital, Cheeloo College of Medicine, Shandong University, 324 Jingwu Road, Jinan 250021, Shandong, People's Republic of China.

Received: 10 May 2020 Accepted: 2 November 2020

Published online: 16 November 2020

\section{References}

1. Biddle SJ, Garcia Bengoechea E, Wiesner G. Sedentary behaviour and adiposity in youth: a systematic review of reviews and analysis of causality. Int J Behav Nutr Phys Activity. 2017;14(1):43.

2. Boeckxstaens GE, de Jonge WJ. Neuroimmune mechanisms in postoperative ileus. Gut. 2009;58(9):1300-11.

3. Luckey A, Livingston E, Tache Y. Mechanisms and treatment of postoperative ileus. Arch Surg (Chicago, III: 1960). 2003;138(2):206-14.

4. Wehner S, Vilz TO, Stoffels B, Kalff JC. Immune mediators of postoperative ileus. Langenbeck's Arch Surg. 2012;397(4):591-601.

5. Resnick J, Greenwald DA, Brandt LJ. Delayed gastric emptying and postoperative ileus after nongastric abdominal surgery: part I. Am J Gastroenterol. 1997;92(5):751-62. 
6. Gunduz $\mathrm{OH}$, Kenis-Coskun O. Ganglion blocks as a treatment of pain: current perspectives. J Pain Res. 2017;10:2815-26.

7. Zhao HY, Yang GT, Sun NN, Kong Y, Liu YF. Efficacy and safety of stellate ganglion block in chronic ulcerative colitis. World J Gastroenterol. 2017;23(3):533-9.

8. Yokoyama M, Nakatsuka H, Itano Y, Hirakawa M. Stellate ganglion block modifies the distribution of lymphocyte subsets and natural-killer cell activity. Anesthesiology. 2000;92(1):109-15.

9. Yang X, Shi Z, Li X, Li J. Impacts of stellate ganglion block on plasma NF-kappaB and inflammatory factors of TBI patients. Int J ClinExp Med. 2015;8(9):15630-8.

10. Moher D, Liberati A, Tetzlaff J, Altman DG. Preferred reporting items for systematic reviews and meta-analyses: the PRISMA statement. PLoS Med. 2009;6(7):e1000097.

11. Higgins JP, Thompson SG, Deeks JJ, Altman DG. Measuring inconsistency in meta-analyses. BMJ (Clinical research ed). 2003;327(7414):557-60.

12. Chunying Z, Jingfang L, Meizheng $Y$, Ting Z, Xiaochun Z. Effect of ultrasound-guided stellate ganglion block on gastrointestinal function in patients undergoing gynecological laparoscopic operation. Med Innov China. 2019;16(15):9-13.

13. Lihua C, Ya W, Shuijing W, Hui Y, Hui L, Yaping L, Haihong W, Xiangming F, Guohao X. Effect of ultrasound-guided stellate ganglion block on perioperative inflammatory responses and postoperative recovery of gastrointestinal function in patients undergoing gastrointestinal surgery. Chin J Anesthesilol. 2018;38(8):904-7.

14. Yuxin S, Xizhe Z, Qi Z, Xiaodong L, Jiannan S, Haaibo L. Effects of stellate ganglion block combined with epidural and general anesthesia on the rapid recovery for patients after radical gastrectomy for gastric cancer. Beijing Med J. 2018:40(9):854-7.

15. Renbo S, Yaohua W, Fude S, Zuen T. Effects of stellate ganglion block on the stress response and postoperative recovery in patients with gastrointestinal tumors. J Clin Med Pract. 2013;21:175-7.

16. Ke P, Juan Z, Wei-Rong C, Hua-Yue L, Fu-Hai J. Ultrasound-guided stellate ganglion block improves gastrointestinal function after thoracolumbar spinal surgery. ClinTherap. 2017:39(11):2322-30.

17. Sterne JAC, Savović J, Page MJ, Elbers RG, Blencowe NS, Boutron I, Cates CJ, Cheng HY, Corbett MS, Eldridge SM, et al. RoB 2: a revised tool for assessing risk of bias in randomised trials. BMJ (Clinical Research ed) 2019;366:14898.

18. Venara A, Neunlist M, Slim K, Barbieux J, Colas PA, Hamy A, Meurette G. Postoperative ileus: pathophysiology, incidence, and prevention. J ViscerSurg. 2016;153(6):439-46.

19. HedrickTL, McEvoy MD, Mythen MMG, Bergamaschi R, Gupta R, Holubar SD, Senagore AJ, Gan TJ, Shaw AD, Thacker JKM, et al. American Society for enhanced recovery and perioperative quality initiative joint consensus statement on postoperative gastrointestinal dysfunction within an enhanced recovery pathway for elective colorectal surgery. AnesthAnalg. 2018;126(6):1896-907.
20. Popping DM, Elia N, Van Aken HK, Marret E, Schug SA, Kranke P, Wenk M, Tramer MR. Impact of epidural analgesia on mortality and morbidity after surgery: systematic review and meta-analysis of randomized controlled trials. Ann Surg. 2014;259(6):1056-67.

21. Lohsiriwat V. Opioid-sparing effect of selective cyclooxygenase-2 inhibitors on surgical outcomes after open colorectal surgery within an enhanced recovery after surgery protocol. World I GastrointestOncol. 2016;8(7):543-9.

22. Kim SM, Youn HG, An JY, Choi YY, Noh SH, Oh SJ, Sohn TS, Kim S. Comparison of open and laparoscopic gastrectomy in elderly patients. J GastrointestSurg. 2018;22(5):785-91.

23. Boelens PG, Heesakkers FF, Luyer MD, van Barneveld KW, de Hingh IH, Nieuwenhuijzen GA, Roos AN, Rutten HJ. Reduction of postoperative ileus by early enteral nutrition in patients undergoing major rectal surgery: prospective, randomized, controlled trial. Ann Surg. 2014;259(4):649-55.

24. van den Heijkant TC, Costes LM, van der Lee DG, Aerts B, Osinga-de Jong M, Rutten HR, Hulsewe KW, de Jonge WJ, Buurman WA, Luyer MD. Randomized clinical trial of the effect of gum chewing on postoperative ileus and inflammation in colorectal surgery. Br J Surg. 2015;102(3):202-11.

25. Luthra P, Burr NE, Brenner DM, Ford AC. Efficacy of pharmacological therapies for the treatment of opioid-induced constipation: systematic review and network meta-analysis. Gut. 2018;3:434-44.

26. Jin W, Li Q, Luo X, Zhong J, Song Y, Li Y. Da-cheng-qi decoction combined with conventional treatment for treating postsurgical gastrointestinal dysfunction. Evid Based ComplemAltern Med eCAM. 2017;2017:1987396.

27. Raut MS, Maheshwari A. Stellate ganglion block: important weapon in the anesthesiologists' armamentarium. J CardiothoracVascAnesth. 2018:32(2):e36-7.

28. Narouze S. Ultrasound-guided stellate ganglion block: safety and efficacy. Curr Pain Headache Rep. 2014;18(6):424.

29. Haaga JR, Kori SH, Eastwood DW, Borkowski GP. Improved technique for CT-guided celiac ganglia block. AJR Am J Roentgenol. 1984;142(6):1201-4.

30. Feigl GC, Rosmarin W, Stelzl A, Weninger B, Likar R. Comparison of different injectate volumes for stellate ganglion block: an anatomic and radiologic study. RegAnesth Pain Med. 2007;32(3):203-8.

31. Hardy PA, Wells JC. Extent of sympathetic blockade after stellate ganglion block with bupivacaine. Pain. 1989;36(2):193-6.

32. Jiang W, Zheng B. Stellate ganglion block. Pain Clin J. 2007;3(2):106-14.

\section{Publisher's Note}

Springer Nature remains neutral with regard to jurisdictional claims in published maps and institutional affiliations. 